\title{
Resource Allocation for Optical Packet-switching Routers with Self-similar Bursty Traffic
}

\author{
J. J. He and D. Simeonidou \\ Dept. of Electronic Systems Engineering, University of Essex, Colchester CO4 3SQ, UK
}

Key words: self-similarity, packet-switching routers, optical buffering, wavelength conversion, multi-path routing

\begin{abstract}
This paper addresses the problem of resource allocation in optical packetswitching routers under self-similar bursty Internet traffic conditions. The contention resolution techniques such as optical buffering and wavelength conversion are considered. The router performance is evaluated in terms of packet loss probability and buffer overflow probability via numerical simulations. Results show that wavelength conversion is a promising technique in improving the router performance. Our findings suggest that selfsimilar traffic significantly increases the resource requirement compared with similar studies performed using traditional bursty traffic models.
\end{abstract}

\section{INTRODUCTION}

It is believed that Internet packet traffic will dominate telecommunication network capacity in the near future due to the widely used TCP/IP protocol suite [1]. Consequently packet-switching routers (PSRs) in the network are expected to efficiently handle high throughputs of over $1 \mathrm{Tbit} / \mathrm{s}$. With the development of state-of-the-art optical technology, it is possible to implement the high-throughput PSRs in the optical domain [2].

In designing an optical packet-switching router (OPSR) resource allocation is a critical issue to achieve a good router performance in a costeffective manner, in terms of packet loss probability (PLP), buffer overflow probability (BOP), packet delay and throughput. Network resources include optical buffers, wavelength converters, tunable lasers, etc. Among them the 
Optical buffers comprising optical delay lines are crucial and integral parts of the OPSRs. Due to the fact that light travels on-the-fly and can't be removed from the buffer till the end of the buffering period, optical buffers lack flexibility comparing with electronic ones [3]. Moreover the complexity of OPSRs increases with the increase of the number of fiber delay-lines; thus reduction to the number of optical buffers is preferable.

Wavelength converters are essential in improving the efficiency of wavelength-division multiplexed (WDM) networks for both circuitswitching [4] and packet-switching [5] schemes. All-optical wavelength conversion is receiving much attention; the main techniques include fourwave mixing (FWM), difference frequency generation (DFG), cross-gain modulation (XGM) and cross-phase modulation (XPM) in semiconductor optical amplifiers [6]. An ideal wavelength converter should possess such characteristics as transparency to bit rates and signal formats, fast setup time of output wavelength, conversion to both shorter and longer wavelengths, insensitivity to input signal polarization, simple implementation, and so on.

Investigations on resource allocation were reported in the literature [5][7], most of which were based on Markovian assumptions where the packet arrivals were typically modeled by random processes that decay with light-tails, e.g. Poisson distribution, leading to short-range dependence (SRD). As a result the router performance can be improved significantly (i.e. exponential decay rate) by slightly increasing the resources. For example the PLP of a router, with an average packet length of 2 , a load per channel of 0.4 and 18 buffers, was decreased from $10^{-6}$ to $10^{-10}$ with buffers increasing to 32 , or to $10^{-11}$ by employing 2 wavelength conversions [5].

On the contrary, recent studies on traffic traces from packet-based localarea and wide-area networks indicated that Internet traffic is characterized by random process decaying with heavy-tail [8][9]. This results in a longrange dependent (LRD) process that statistically exhibits self-similarity. The self-similar traffic is bursty on a wide range of time scales, a much wider range than that is captured by traditional traffic models.

Since then a lot of work has been carried both on constructing models capable of generating synthetic traffic with matching characteristics with self-similar process [8][9] and on evaluating the effect of self-similar traffic on network performance [10]. Most of the performance evaluations were based on ATM networks, where the cell loss probability and the buffer overflow probability were analytically shown decreasing no more than hyperbolically under LRD process but decreasing exponentially under traditional traffic process [10]. These results strongly suggest that there exist necessities to control the self-similarity in the network layer or higher layers and to deal with the self-similar traffic in the physical layer with more careful manner. Recently some of the protocols in the network layer or 
higher were reported to control the self-similar traffic. In [11] a selective aggressiveness control was adopted for on-line detecting the long-range dependence and consequently improving the throughput. In [12] selected large traffic bursts were shaped at the local access node, which was shown to be capable of decreasing ATM cell loss at a bottleneck queue at the expense of increasing the transmission time of a few large files. However the efficiencies of these methods are to be further refined. On the other hand, there is very little work reported in dealing with self-similar traffic in the lower layers, particularly in the optical packet layer.

In this paper we investigate the resource allocation for optical packetswitching routers conveying self-similar bursty traffic. Optical buffers and wavelength converters are considered as the main network resources. Performance study of OPSRs regarding PLP and BOP is addressed through numerical simulations. In the next section the traffic model and the simulation environment are described. Simulation results and discussions are presented in section III followed by the concluding remarks in section IV.

\section{SIMULATION ISSUES}

\subsection{Traffic model}

A process has asymptotic second order self-similarity with parameter $H=(3-\alpha) / 2$ if $t^{\alpha} P[\tau>t] \rightarrow z$, as $t \rightarrow \infty, 1<\alpha<2$, (where $\tau$ is the active period of traffic and $z$ is a constant) [10]. The larger the value of $H$, the higher the degree of self-similarity. This characteristic can be well described as a Pareto distribution with cumulative distribution function (CDF) $P[X \leq x]=1-(\theta / x)^{\alpha},(\theta>0)$. When $\alpha \leq 1$ it has infinite mean; $\alpha \leq 2$, infinite variance; and when $\alpha>2$, it belongs to non-LRD region [9]. Our investigations will be based on Pareto distributed random process.

\subsection{Simulation environment}

Traffic process is simulated as a superposition of many sources with Pareto distributed ON and OFF periods [13]. An ON period represents a packet and is always followed by an OFF period for the interval of two consecutive packets. To simplify the simulation, the distribution of $\mathrm{ON}$ and OFF periods has the same degree of self-similarity, which equals to having a channel utilisation of 0.5 , and are bit padded in integral time-unit lengths suitable for transmission and buffering in optical fibers. The $\theta$ in the CDF function signifying the minimum duration generated, is set to 1 in the whole simulation processes. Thus the duration of the ON or OFF period is $\left\lceil U^{-1 / \alpha}\right\rceil$ 
in time unit (where $U$ is a random variable uniformly distributed on $[0,1]$ and $\lceil *\rceil$ indicates the ceiling function).

The generic router architecture is shown in Fig.1, which has equal input and output ports with the same wavelength channels. The core of the router is a non-blocking switching network and is controlled by a central control unit not shown in the figure. Each packet is directed to any output port with equal opportunity. For simplicity a FIFO output buffering is considered, which achieves optimal throughput-delay performance compared to other FIFO buffering schemes [7]. Wavelength converters are placed between the output of the router core and the input of the buffers. With output buffering, when two packets compete for the same output wavelength channel, one of them is buffered and will not be transmitted until the channel location is available. If the buffer is full at that particular moment, the packet is lost and the duration of the packet is labeled as buffer overflow moments. With wavelength conversion, this packet is translated to another wavelength channel at the same output port whose buffer or channel is available, thereby resolving the contention and consequently reducing PLP and BOP. All of the simulations were performed over $1 \times 10^{6}$ time units.

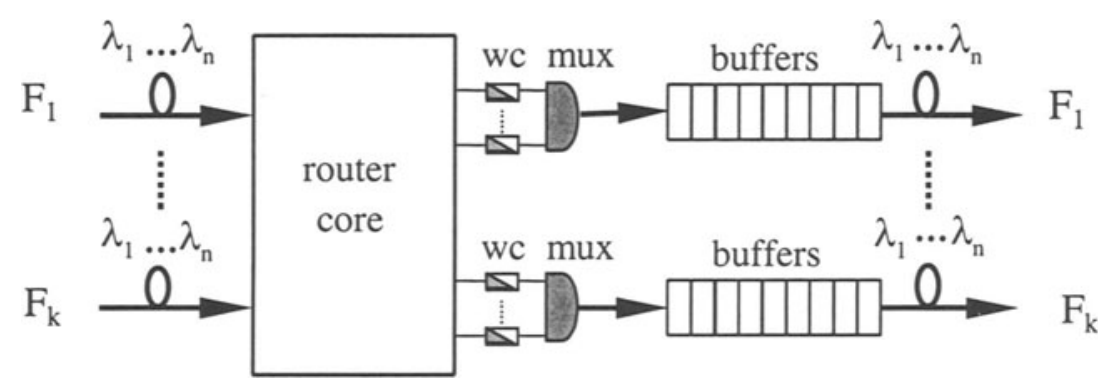

Figure 1. Generic router architecture under simulation

\section{RESULTS AND DISCUSSIONS}

\subsection{Allocation of optical buffers}

Firstly resource allocation for OPSRs with optical buffering is studied. In Fig.2 PLP for a $4 \times 4$ router (i.e. $\mathrm{k}=4$ in Fig. 1) is shown as a function of buffer depth, with $\alpha$-value being used as a parameter. In non-LRD region $(\alpha>2)$ the decay rate of PLP is exponential, which is in good agreement with the results obtained by using traditional traffic models [5]. While in LRD region $(1<\alpha<2)$ the decreasing rate of the PLP is much less than exponential. 
The less the $\alpha$ the heavier the tail of PLP. Thus a large amount of buffers (much more than several tens) will be needed in order to obtain an acceptable PLP of $10^{-10}$ for self-similar traffic in practical networks.

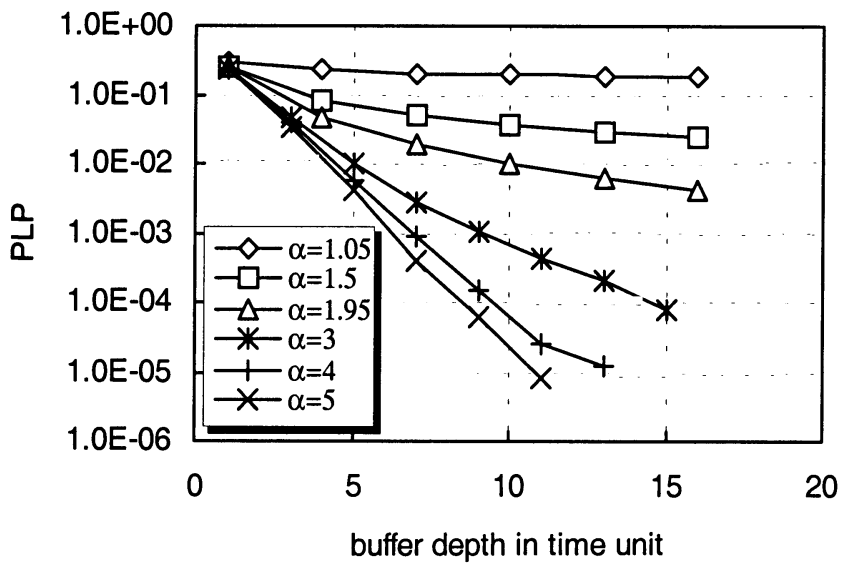

Figure 2. Packet loss probability vs. buffer depth for a $4 \times 4$ router with output buffering and different a-values

The same situation exists when investigating the buffer overflow moments in OPSRs. The analytical BOP for the OPSR is developed as follows: [10]

$$
\text { Poverflow } \geq \frac{c \tau}{\alpha_{\tau}\left(\alpha_{\tau}-1\right)(E \tau+E \kappa)^{2}}\left(\left[\frac{h+b}{a}\right]+2\right)^{-\alpha_{\tau}+1},
$$

where, $c_{\tau}=\alpha_{\tau} \theta_{\tau}^{\alpha_{\tau}}=\left(\sum_{n=1}^{\infty}{ }^{n^{-\alpha_{\tau}-1}}\right)^{-1}$ for ON period distribution $\left\{\tau_{i}\right\}$, and $E_{\tau}=c_{\tau} \sum_{n=1}^{\infty} n^{-\alpha_{\tau}} ; c_{\kappa}=\alpha_{\kappa} \theta_{\kappa}^{\alpha_{\kappa}}=\left(\sum_{n=1}^{\infty} n^{-\alpha_{\kappa}-1}\right)^{-1}$ for OFF period distribution $\left\{\kappa_{i}\right\}$, and $E_{\kappa}=c_{\kappa} \sum_{n=1}^{\infty} n^{-\alpha_{\kappa}} ; a=\frac{1}{E_{\tau}+E_{\kappa}}, b=a+1$, and $h$ is the buffer depth for a wavelength channel.

In Fig. 3 the analytical lower bound and the simulation results of BOP are plotted as a function of buffer depth with the number of ports as a parameter for the middle degree of self-similarity $(\alpha=1.5)$. As can be seen that both of the analytical and simulated results of BOP do not decrease faster than hyperbolically with the increase of buffer depth for different routers. In addition, BOP increases but the increase rate slows down as the number of 
port increases. Analytical results show that $10^{9}$ buffers will be needed to obtain a BOP lower than $10^{-5}$ for $\alpha=1.5$.

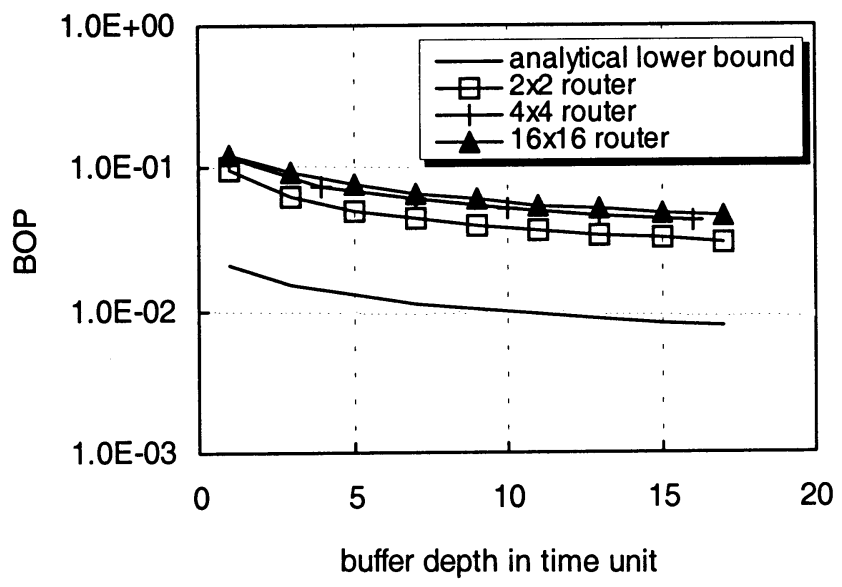

Figure 3. Comparison between simulated and analytical overflow probability for routers with output buffering and $\alpha=1.5$

\subsection{Allocation of wavelength conversions}

Next the resource allocation for routers with wavelength conversion (WC) and optical buffering is investigated. Fig.4 shows PLP as a function of buffer depth for $4 \times 4$ router with self-similar traffic of $\alpha=1.5$. It can be observed that PLP decreases with the deployment of WC. Moreover the use of WC changes the heavy tail distribution of the PLP, which shows itself a promising resource for the future OPSRs.

To further verify this point the PLP versus the number of WCs is depicted in Fig. 5 for a $4 \times 4$ router with 16, 50, 100, 300 buffers respectively. The super-linear projection lines corresponding to different amount of buffer depth are plotted in Fig. 5 as well. As can be seen that PLP decreases two orders in magnitude for the case of 6 WCs and 50 buffers comparing with the case of 300 buffers only, and decreases one order in magnitude comparing with that of $3 \mathrm{WCs}$ and 100 buffers. It can be expected that as the number of WCs increases the PLP decreases. The rate of decrease strongly depends on the buffer depth. As evidence, with 300 buffers the PLP decreases much faster than that with 16 buffers. The projection line for the case of 300 buffers indicates that to obtain the PLP of $10^{-10}$ there will need 12 wavelength conversions. Compared with traditional traffic models, the resources required under LRD traffic model are much greater than the case of traditional SRD traffic. 


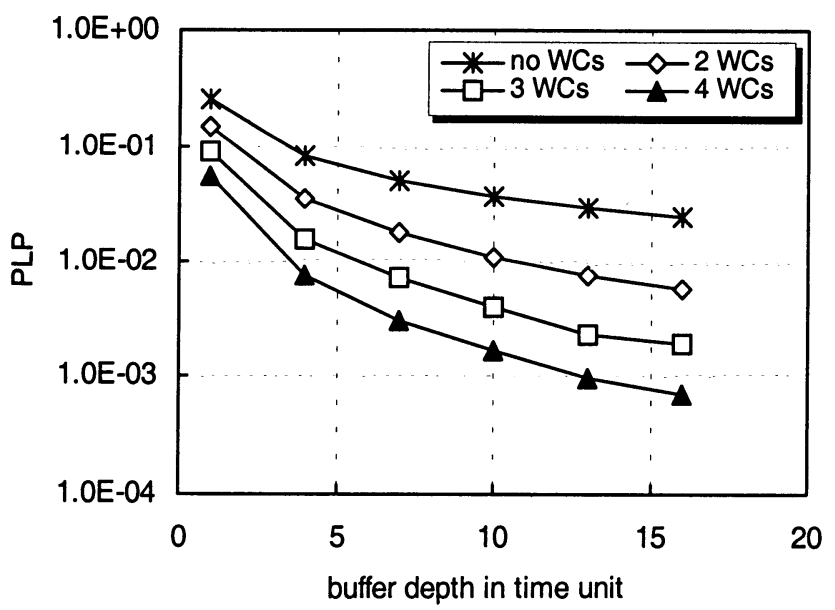

Figure 4. Packet loss probability vs. buffer depth for a $4 \times 4$ router with wavelength conversion and $\alpha=1.5$

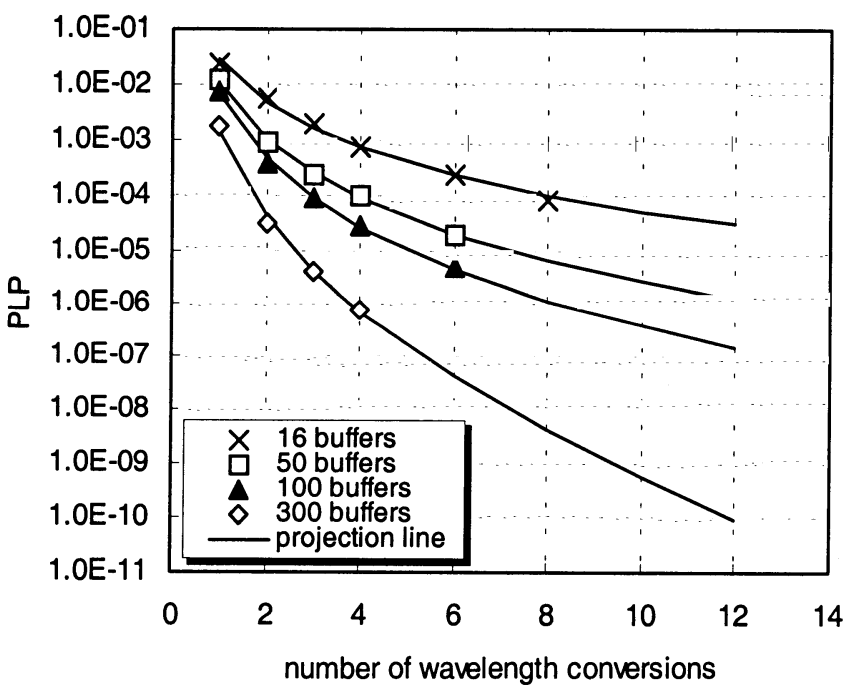

Figure 5. Packet loss probability vs. number of wavelength conversions for a $4 \times 4$ router with different buffer depth and $\alpha=1.5$

\section{CONCLUSIONS}

A model describing the self-similarity of Internet traffic has been used in investigating the resource allocation for optical packet-switching routers. The router performance has been evaluated through simulations. It has been 
shown that optical buffering on its own could not offer adequate router performance. The addition of wavelength conversions resulted in a decrease of the heavy tail distribution of packet loss probability and thus a better router performance. Our work strongly suggests that in comparison with traditional traffic models the self-similar traffic does heavily influence the resource requirement in the router.

Acknowledgements: The authors wish to thank Corning Optical Fibres for funding this research project.

\section{References}

[1] S. A. Thomas, Ipng and the TCP/IP protocols implementing the next generation Internet, John Wiley \& Sons, Inc., 1996

[2] M. Renaud, F. Masetti, C. Guillemot and B. Bostica, "Network and system concepts for optical packet switching", IEEE Communications Magazine, April 1997, pp. 96-102

[3] D.K. Hunter, M.C. Chia and I. Andonovic, "Buffering in optical packet switches", Journal of Lightwave Technology, 1998, vol. 16, pp. 2081-2094

[4] R.A. Barry and P.A. Humblet, "Models of blocking probability in all-optical networks with and without wavelength changers", IEEE Journal on Selected Areas in Communications, 1996, Vol. 14, pp. 858-867

[5] S. L. Danielsen, C. Joergensen, B. Mikkelsen, and K. E. Stubkjaer, "Analysis of a WDM packet switch with improved performance under bursty traffic conditions due to tuneable wavelength converters", Journal of Lightwave Technology, 1998, vol. 16, pp. 729-735

[6] B. Ranamurthy and B. Mukherjee, "Wavelength conversion in WDM networking", IEEE Journal on Selected Areas in Communications, 1998, vol. 16, pp. 1061-1073

[7] M. G. Hluchyj and M. J. Karol, "Queueing in high-performance packet switching", IEEE Journal on Selected Areas in Communications, 1988, vol. 6, pp. 1587-1597

[8] W. E. Leland, M. S. Taqqu, W. Willinger and D. V. Wilson, "On the self-similar nature of Ethernet traffic (extended version)", IEEE/ACM Transactions on Networking, 1994, vol. 2, pp. $1-15$

[9] V. Paxson and S. Floyd, "Wide area traffic: the failure of Poisson modeling", IEEE/ACM Transactions on Networking, 1995, vol. 3, pp. 226-244

[10] B. Tsybakov and N. D. Georganas, "On self-similar traffic in ATM queues: definition, overflow probability bound and cell delay distribution", IEEE/ACM Transactions on Networking, 1997, vol. 5, pp. 397-408

[11] T. Tuan and K. Park, "Multiple time scale congestion control for self-similar network Traffic", Performance Evaluation, 1999, vol. 36 7, pp. 359-386

[12] K.J. Christensen and V. Ballingam, "Reduction of self-similarity by application-level traffic shaping", $22^{\text {nd }}$ IEEE Conference on Local Computer Networks, pp. 511-518, Nov. 1997

[13] L. S. Tamil, F. Masetti, T. McDermott, G. Castanon, An Ge and L. Tančevski, "Analysis of all-optical IP routers", SPIE Conference on All-optical Networking, pp. 456-464, Boston, Nov. 1998

[14] G. A. Castañón, O. K. Tonguz and A. Bononi, "Benefits of wavelength translation in datagram all optical networks", Electronics Letters, 1997, vol. 33, pp. 1567-1568 